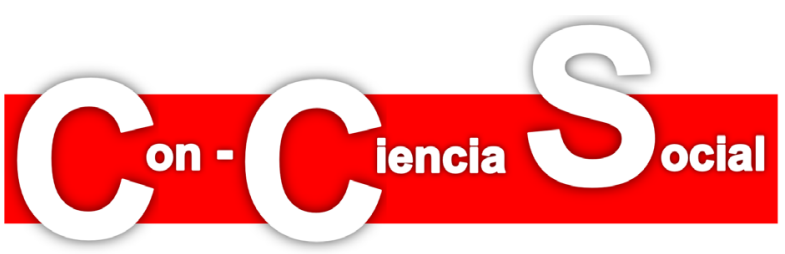

\title{
La Historia de los conceptos para una ciudadanía crítica. Un proyecto de transferencia de la Begriffgeschichte al ámbito educativo
}

\section{The history of concepts for critical citizenship. A project to transfer the Begriffgeschichte to the educational field}

\author{
Aurora Rivière \\ Universidad Complutense de Madrid \\ ariviere@ucm.es
}

Recibido en agosto de 2019

Aceptado en noviembre de 2019

DOI:10.7203/con-cienciasocial.3.16796

\section{RESUMEN}

En estas páginas se presenta un proyecto de innovación docente que se desarrolla en la Universidad Complutense de Madrid sobre la transferencia de la Historia de los conceptos al ámbito de la enseñanza, destacando el interés de esta transposición para la formación de ciudadanías críticas. Se exponen, así mismo, los fundamentos teóricos y metodológicos que sirven de guía para llevar a cabo esta transferencia.

Palabras clave: historia de los conceptos, transposición didáctica, educación para la ciudadanía, enseñanza de la historia.

\begin{abstract}
In these pages, an educational innovation project developed at the Complutense University of Madrid based on the transfer of the History of concepts to the field of education is introduced, highlighting the interest of this transposition for the education of critical citizenship. The theoretical and methodological foundations that serve as a guide to conduct this transfer are also discussed below.
\end{abstract}

Keywords: history of concepts, didactic transposition, education for citizenship, teaching of history.

\section{Referencia}

Rivière, A. (2020). La Historia de los conceptos para una ciudadanía crítica. Un proyecto de transferencia de la Begriffgeschichte al ámbito educativo. Con-Ciencia Social (segunda época), 3, 175-184. DOI:10.7203/con-cienciasocial.3.16796 
Aurora Rivière, La Historia de los conceptos para una ciudadanía crítica...

Manifestaba Reinhart Koselleck, padre de la denominada Historia de los conceptos (Begriffgeschichte), la imposibilidad de tratar rigurosamente la historia sin aclararse respecto a las categorías en virtud de las cuales se va a expresar (Koselleck, 1993) y destacaba la necesidad de reparar en la historicidad de los conceptos políticos y sociales y en su relación (siempre compleja) con las transformaciones que tienen lugar en la sociedad. La propuesta del "historiador pensante" -según le denominaba su maestro Gadamer- resulta particularmente sugerente al plantearnos la enseñanza de la historia escolar, claramente orientada hacia el mantenimiento y la naturalización de un orden imperante en el que el manejo espurio de determinados conceptos juega un papel fundamental.

Conscientes de ello, así como de la gran complejidad de los conceptos políticos y sociales que manejamos, de las dificultades que presentan los estudiantes para su comprensión y de la escasa e inadecuada atención didáctica que reciben, nos planteamos la necesidad de incorporar su historia en la enseñanza tomando como referencia diferentes propuestas surgidas con el Linguistic turrn. Sobre todo de mano de la Begriffgeschichte de Reinhart Koselleck, pero considerando también diferentes aportaciones de la Escuela de Cambridge -de Quentin Skinner, J.G.A. Pocock y John Dunn entre otros-así como del History of Political and Social Concept Group vinculado a ella y fundado a iniciativa de Melvin Richter. Trataremos de exponer someramente en estas páginas las principales premisas teóricas y metodológicas de estas perspectivas como punto de partida para llevar a cabo su transferencia al ámbito escolar, considerando su interés para la formación de ciudadanos conscientes, adiestrados en el análisis crítico de conceptos y discursos.

A pesar de las distancias que separan a los historiadores del pensamiento político congregados en torno a la Escuela de Cambridge de los postulados de la historia conceptual de Reinhart Koselleck, en ambos encontramos una reacción y crítica a la tradicional Historia de las ideas, concebidas como constructos estáticos atemporales y eternos de validez universal y, por ello, dotados de una continuidad de sentido que trasciende lo histórico. Esta consideración implicaría el manejo de un repertorio conceptual sustancialmente unitario cuya interpretación sería homogénea, ya que se le concede a la idea un sentido estable y análogo a lo largo del tiempo. Es la concepción que manifiestan los docentes cuando solicitan a los estudiantes "definiciones" o glosarios de conceptos, como si fuera algo que pudiera realizarse al margen de su contexto de validez y de su uso temporal concreto. En contraste con las ideas concebidas como proposiciones universales de carácter perenne, los conceptos 
Aurora Rivière, La Historia de los conceptos para una ciudadanía crítica...

poseen para Koselleck una historicidad inherente: adquieren significado en la historia. El significado no se le atribuye a una entidad que permanece estática -a modo de los ideales tipo weberianos-, sino que se construye en relación a su espacio de validez en un contexto histórico determinado, en función de su específica situación temporal. Es decir, los conceptos también tienen una historia, se crean, transforman y modifican como parte del cambio político y social.

Historizar y deconstruir los conceptos políticos y sociales que manejamos implica, por eso, despojarlos de su apariencia estática y "natural" y subrayar el carácter contingente y precario de sus significados, lo que constituye una premisa esencial si pretendemos abordar una enseñanza crítica de la historia y propulsar la autoconciencia política en los jóvenes estudiantes. Implica, así mismo, una toma de conciencia, por parte de historiadores y docentes, de las herramientas conceptuales de las que se sirven al reconstruir o enseñar la historia y de los motivos por los que se utilizan unos determinados conceptos en lugar de otros.

$\mathrm{Si}$ un concepto no es equiparable a una idea en su concepción tradicional, tampoco es para Koselleck lo mismo que una palabra. El concepto posee una densidad semántica que no tiene una palabra cualquiera. Tanto la palabra como el concepto son polisémicos, pero de distinta manera. Mientras que la polisemia de las palabras es eventual -su significado puede depender del contexto lingüístico y de la situación en que es utilizada-, el concepto es estructuralmente polisémico. En su uso, una palabra puede ser unívoca pero el concepto mantiene siempre su polivocidad. En ellos se va tejiendo una especie de red semántica que aglutina una pluralidad de significaciones imbricadas entre sí, que van conformando cierta unidad de sentido. Concentran y sintetizan una pluralidad de significados de manera compleja y en ocasiones polémica. Esa concentración de contenidos semánticos procede, según Koselleck, de la necesidad de expresar la diversidad de la experiencia histórica, por eso su significado no puede determinarse al margen de su uso en la sociedad.

Para Koselleck, los conceptos tampoco son meras representaciones de un estado de cosas, un simple reflejo de una realidad política o social. Un concepto es indicador, pero también factor de la realidad, recoge y crea un contexto determinado, "reúne la pluralidad de la experiencia histórica y una suma de relaciones teóricas y prácticas en un contexto que... solo está dado y se hace experimentable por el concepto" (Koselleck, 1993, p. 117). Aúnan la doble condición de producto y productor de realidades, no son simples testigos, son agentes a su vez. Registran y acuñan a la vez propulsan y generan. De ahí su naturaleza dual, lingüística y extra-lingüística 
simultáneamente (que es la que une a la Begriffgeschichte con la historia social), y de ahí también su carácter, en ocasiones, polémico.

Todo proceso de significación conlleva implícitamente otro de hegemonización, de tal forma que determinados conceptos se inscriben en una pugna semántica que forma parte de las propias luchas sociales y políticas. Alguno de los conceptos políticos y sociales fundamentales (aquellos que concentran una mayor densidad semántica) no son portadores de significados incontrovertibles, lo que sugiere, como afirma Melvin Richter, que el significado de algunos conceptos deriva más bien de la controversia que del consenso. "Ciertos conceptos son valiosos no a pesar de las disputas sobre su significado, sino precisamente debido a tales discrepancias" (Richter, 2003, p. 462). De esta forma la oposición y el desacuerdo se presentan como aspectos esenciales en los procesos de significación de algunos conceptos.

Desde la Escuela de Cambridge se insiste en la dimensión pragmática del lenguaje -en su uso- al interpretar los lenguajes políticos y los conceptos que los integran como actos y poner el énfasis en el carácter performativo de ese tipo de lenguajes. En el lenguaje performativo mediante la oración y el habla se ejecuta una acción que va mucho más allá del mero acto de decir, se utilizan las palabras para actuar en y sobre la realidad. Quentin Skinner, uno de los más insignes representantes de la Escuela, parte de las tesis del filósofo de la Universidad de Oxford John Langshaw Austin, que diferenciaba dos tipos de oraciones: constatativas (en las que se describe un estado de cosas) y realizativas o performativas, en las que se actúa al decir, no se describe nada, sino que se hace lo que se dice (Austin, 1962). Austin incorporaba más tarde como unidad de análisis y comunicación los actos de habla, que integran -según el filósofo- tres acciones diferentes: el acto locutorio (el acto de referir, de relatar...), el acto ilocutorio (el acto de hacer al decir) y el acto perlocutorio (los efectos que se pretenden sobre la audiencia al realizar el acto). Posteriormente, John Searle llevaba a cabo una reformulación y sistematización de las tesis de Austin en su libro Actos de habla (Searle, 1969).

Los actos ilocutorios y perlocutorios centran la atención de los historiadores de Cambridge en sus análisis de los discursos políticos: lo que se hace al decir en un debate determinado y lo que se pretende conseguir, la intención, el propósito que se persigue alcanzar de la audiencia con la realización del acto. Para Skinner, se trata de dilucidar la intención de los autores de los textos "conocer las intenciones del autor supone conocer el significado de lo que escribe" (en Bocardo, 2007, p. 122). El análisis de las fuentes debería focalizarse -desde esta perspectiva- en lo que los autores de 
Aurora Rivière, La Historia de los conceptos para una ciudadanía crítica...

los textos estaban haciendo cuando escribían y publicaban sus obras, enmarcando siempre sus palabras en el contexto lingüístico en el que eran dichas y expuestas. La captación de la intencionalidad del autor no puede lograrse exclusivamente estudiando el texto o el contexto en el que se produjo, la única forma de captarla es considerando el texto como un acto intencionado de comunicación entre el autor y el público al que iba dirigido. Por lo tanto, se trataría de interpretar los textos políticos como actos de habla de sus autores, elaborados en relación con el contexto en que se producen, en el que las convenciones lingüísticas vigentes y las audiencias sobre las que el autor quiere actuar tienen un interés especial. Actos y efectos perlocutivos -y en menor medida los ilocutivos- constituirían, pues, la esencia de los textos políticos, y es lo que debe preocupar -desde esta perspectiva- en un análisis conceptual.

La historia de los conceptos no puede obviar los aspectos referentes a la pragmática, sin los que quedaría absolutamente desvirtuado cualquier análisis conceptual, pero tampoco tiene que limitarse al análisis de esos aspectos. El estudio de los procesos históricos de significación y resignificación de conceptos es indisociable del de sus usos concretos.

Desde el punto de vista metodológico, la Begrisffsgeschichte de Reinhart Koselleck aporta instrumentos útiles y de gran interés para abordar el estudio y la enseñanza de los conceptos. La Historia de los conceptos es, ante todo, para el historiador alemán, una herramienta metodológica imprescindible para el análisis de las fuentes históricas. La Begrisffsgeschichte se ofrece pues como tarea esencialmente historiográfica, un método especializado en la crítica de fuentes históricas y por tanto una herramienta hermenéutica de la investigación histórica que se ocupa de la formación de los conceptos, de sus usos y modificaciones en el tiempo, con la que se posibilita el esclarecimiento de procesos políticos y sociales. La Historia de los conceptos se presenta por ello como metodología de análisis de las fuentes que incita a un ejercicio hermenéutico e invita a la reflexión sobre procesos históricos complejos.

En este sentido, constituye un antídoto contra lecturas esencialistas y "objetivas" de las fuentes, que a menudo se presentan e interpretan en los textos escolares como testimonios dotados de una verdad esencial y que muestran a los estudiantes, a modo de espejo, la realidad a la que hacen referencia de una manera pretendidamente objetiva. Frente a ello, tanto la Begrisffsgeschichte como la Historia del Pensamiento Político de Cambridge nos las presentan en su contingencia y temporalidad, como 
Aurora Rivière, La Historia de los conceptos para una ciudadanía crítica...

constructos y constructoras de una realidad concreta, inscritas en el marco de un determinado debate social y político en el que se producen como actos -según subrayan Quentin Skinner o J.G.A. Pocock- señalados por una carga de intencionalidad y limitados por los marcos lingüísticos de la época.

Entre las herramientas metodológicas que nos aporta la Begrisffsgeschichte nos parecen particularmente útiles para la comprensión de la historicidad de los conceptos la combinación analítica sincronía/diacronía y las categorías de espacio de experiencia y horizonte de expectativas con las que Koselleck elabora su ingeniosa teoría de los tiempos históricos y explica los elementos de permanencia, cambio o innovación de los conceptos a lo largo de su historia.

Para analizar los conceptos Koselleck propone proceder desde una doble perspectiva, sincrónica y diacrónica, ambas integran su historia. Los conceptos se significan y aparecen insertos en un contexto de sentido, como parte de una realidad en la que intervienen. Acumulan una diversidad de experiencias que caracterizan a ese contexto específico. Esta es la dimensión sincrónica del concepto. Pero para elaborar su historia es preciso liberar a los conceptos de su contexto situacional y examinarlos en su sucesión temporal. Se tendrán en cuenta las diferentes significaciones y usos que los conceptos han ido manteniendo o perdiendo a lo largo del tiempo y la forma en que se relacionan esas diferencias con aquello que sucedía. Esta es la dimensión diacrónica, que adopta la perspectiva de la larga duración e intenta detectar los diferentes estratos temporales del concepto. A través de esta perspectiva puede establecerse la permanencia, el cambio o la novedad de los conceptos. Para que sea posible este análisis diacrónico, es necesaria una referencia al presente, una traducción al lenguaje actual a través de conceptos estructurales diferentes a los que se usaron en un período particular. Lo contrario, afirma Koselleck, sería lo mismo que una edición de las propias fuentes.

La Begrisffsgeschichte de Koselleck utiliza dos categorías de gran potencialidad heurística, de naturaleza no histórica sino antropológica: el espacio de experiencia y el horizonte de expectativas. Constructos que derivan de la experiencia humana misma - de la memoria y la utopía- y que permiten detectar los cambios y la estabilidad de los conceptos en el largo plazo. La experiencia hace referencia al pasado presente, es decir, a lo que pervive en los recuerdos, racionalizaciones y formas de conducta que han sido transmitidas de una generación a otra. La expectativa alude al futuro presente, a las proyecciones de futuro que se contemplan, o al futuro pasado, las que se contemplaron en otras épocas. Teniendo en cuenta la manera en que los conceptos 
Aurora Rivière, La Historia de los conceptos para una ciudadanía crítica...

integran experiencias y expectativas se pueden detectar cambios estructurales durante largos períodos de tiempo.

Son las dos categorías con las que tematiza el tiempo histórico -en el que se entrecruzan el pasado, el presente y el futuro- y serán las que utilice para desarrollar la tesis central de su obra magna (Geschichtliche Grundbegriffe) en relación al supuesto del Sattelzeit. Se trata del período de la historia europea (que abarcaría aproximadamente entre 1750 y 1850), en el que -entre los fenómenos que acompañan a la modernidad- se suceden un conjunto de transformaciones fundamentales en el léxico político y social en el que veremos cómo se produce en los conceptos un distanciamiento entre el espacio de la experiencia y el horizonte de expectativas, una mutación conceptual en la que irán perdiendo el contenido vivencial (contra el que surgían) muchos conceptos, que se futurizaban en relación con los profundos cambios y transformaciones que se experimentaban en la sociedad. Cargados de expectativas y con una clara orientación de movimiento, veremos aparecer entonces un buen número de conceptos acabados en ismo que apuntan hacia escenarios de futuro (liberalismo, socialismo, comunismo, anarquismo, nacionalismo, patriotismo...) o veremos imponerse otros singularizados y despojados de sus antiguos usos en plural, transformados por este medio en fórmulas naturalizadas de consenso (Estado, Historia, Nación, Progreso, Revolución, Civilización, Cultura...), con las que se iban modelando las realidades a las que hacían referencia. Algunos conceptos que formaban parte anteriormente de un estamento determinado se democratizan y empiezan a ser compartidos por otros miembros de la sociedad -ampliándose de esta forma el ámbito de parlantes y de usos del conceptocomo ocurre con el de educación. A la vez otros, característicos de la sociedad estamental, comenzaban a caer en desuso, como sucedería con el concepto de súbdito, al que venía a reemplazar el de ciudadano en referencia a las nuevas realidades socio políticas. Aparecen neologismos que expresan nuevos proyectos políticos: los conceptos dejan de referirse a lo conocido -al espacio de la experienciay empiezan a designar estados de cosas aún no vividos, horizontes de expectativas. La tesis del Sattelzeit, tan cuestionada como encomiada por distintos historiadores, nos proporciona una perspectiva interesante desde la que contemplar la gestación o resignificación de muchos de los conceptos que forman parte del lenguaje político y social contemporáneo.

Considerando estas premisas -sincronía/diacronía, espacio de experiencia/horizonte de expectativas- nos hemos planteado los parámetros 
Aurora Rivière, La Historia de los conceptos para una ciudadanía crítica...

metodológicos que sirven de guía a nuestra propuesta de análisis y enseñanza de los conceptos políticos y sociales. Para ello hemos considerado las sugerencias de Arno J. Mayer recogidas por Enzo Traverso (2016) a modo de "reglas" que exponemos a continuación. La primera de estas reglas es la de la contextualización, que implicará la situación del concepto en el marco social, político y lingüístico de la época en que surge y se utiliza en relación a una situación histórica concreta, abordándolo, por tanto, desde la perspectiva de la sincronía. La segunda regla es la de la historización (la del historicismo dice Traverso), o la de la temporalización si se quiere, que implica la necesidad de adoptar una mirada diacrónica para captar la evolución en los usos y significados del concepto a lo largo del tiempo, aplicando la perspectiva braudeliana de la larga duración. La tercera regla es la comparación, de conceptos y contextos, puesto que -como indica Traverso- comparar una idea es un medio indispensable para establecerla, conocerla y utilizarla. Añadimos una cuarta regla en precisión de la anterior, la de la contraposición, de utilidad en referencia al momento de gestación de la significación del concepto, en contraste y oposición a contextos anteriores y a otros conceptos. Su perspectiva es, por tanto, la sincrónica, a diferencia de la comparación que permite adoptar miradas diacrónicas. Finalmente, la conceptualización, desde el presente, para aprehender la realidad a la que hacemos referencia, sin olvidar jamás, como dice Traverso "que la historia real no coincide con sus representaciones abstractas" (Traverso, 2016, p. 26). Contextualización, historización, comparación, contraposición y conceptualización son los parámetros a partir de los cuales consideramos llevar a cabo el análisis y la enseñanza de los conceptos políticos y sociales.

La comprensión de los conceptos en el marco de las fronteras lingüísticas, políticas y sociales de su época y de su historicidad inherente lleva implícita una crítica a la utilización anacrónica de los conceptos y, con ella, una dimensión política y educativa interesante. Con demasiada frecuencia, si no de manera habitual, escuchamos a políticos, tertulianos o comentaristas de la actualidad utilizar conceptos políticos de manera anacrónica, absolutizándolos y otorgando a los preceptos que contienen un estatus casi natural, una especie de consistencia y de verdad eternas. Este uso anacrónico de los conceptos es una tendencia que podemos observar en muchos productos de amplio consumo cultural. No escapan a él los propios historiadores y profesores de historia. Sin duda, historizar los conceptos invita a una utilización más rigurosa y precisa de ellos. 
Aurora Rivière, La Historia de los conceptos para una ciudadanía crítica...

En última instancia, la historia conceptual puede considerarse un antídoto contra determinados "usos públicos" -utilizando la conocida expresión de Jürgen Habermasde la historia y de los conceptos en torno a los que se articula su explicación y escritura. Ayuda a tomar conciencia a los actores políticos y sociales de la estructura temporal de conceptos similares a aquéllos que guían su acción política, despojándolos de absolutizaciones y naturalizaciones preceptivas. Analizar históricamente los conceptos implica el reconocimiento de la contingencia constitutiva de los lenguajes políticos y de la naturaleza precaria de los significados, previniendo de esta forma contra predicadores de esencias y verdades eternas. Incidir en un estudio de todos esos conceptos absolutizados que orientan la vida práctica de los sujetos es hacerlos conscientes de su estructura temporal y, por tanto, ayudarlos a conducir su actuación cívica de forma más responsable. La historia de los conceptos podría considerarse, de esta manera, como propedéutica de una acción ciudadana y política crítica y reflexiva. Al referir las contingencias y las ambigüedades a las que se han visto sometidos los significados de los conceptos que utilizamos se relativizan sus pretensiones y aumenta la posibilidad de que se utilicen de forma más rigurosa y crítica, también conceptos análogos.

\section{REFERENCIAS}

Austin, J. L. (1998 [1962]). Cómo hacer cosas con palabras. Palabras y acciones. Buenos Aires: Paidós.

Bocardo E. (2007). El giro contextual. Cinco ensayos de Quentin Skinner y seis comentarios. Madrid: Tecnos.

Dunn, J. (1996). La agonía del pensamiento político occidental. Vol. 2. Madrid: Akal.

Koselleck, R. (1993). Futuro pasado: Para una semántica de los tiempos históricos. Madrid: Paidós.

Koselleck, R. (2004). Historia de los conceptos y conceptos de historia, Ayer, 53, 2745.

Koselleck, R. (2012). Historias de conceptos: estudios sobre semántica y pragmática del lenguaje político y social. Madrid: Trotta.

Pocock, J. G. (2002). El momento maquiavélico. Madrid: Tecnos.

Richter, M. (2003). Asignando a la «Begriffsgeschichte» su lugar en la historiografía del pensamiento político, Historia contemporánea, 27, 455-464.

Searle, J. (1980 [1969]): Actos de Habla. Madrid: Cátedra. 
Aurora Rivière, La Historia de los conceptos para una ciudadanía crítica...

Skinner, Q. (1985). Los fundamentos del pensamiento político moderno. México: Fondo de Cultura Económica.

Traverso, E. (2012). La historia como campo de batalla: interpretar las violencias del siglo XX. Buenos Aires: Fondo de Cultura Económica.

Con este propósito se desarrolla el Proyecto de Innovación Docente de la Universidad Complutense de Madrid Diseño de un modelo didáctico para la enseñanza y aprendizaje de los conceptos políticos y sociales (ref. 69/2019). 